\title{
Combining Static and Live Digital Forensic Analysis in Virtual Environment
}

\author{
Sasa Mrdovic, Alvin Huseinovic, Ernedin Zajko \\ Faculty of Electrical Engineering \\ University of Sarajevo \\ Sarajevo, Bosnia and Herzegovina \\ \{smrdovic, ahuseinovic, ezajko\}@etf.unsa.ba
}

\begin{abstract}
Traditional digital forensics is performed through static analysis of data preserved on permanent storage media. Not all data needed to understand the state of examined system exists in nonvolatile memory. Live analysis uses running system to obtain volatile data for deeper understanding of events going on. Sampling running system might irreversibly change its state making collected evidence invalid. This paper proposes combination of static and live analysis. Virtualization is used to bring static data to life. Volatile memory dump is used to enable offline analysis of live data. Using data from memory dump, virtual machine created from static data can be adjusted to provide better picture of the live system at the time when the dump was made. Investigator can have interactive session with virtual machine without violating evidence integrity. Tests with sample system confirm viability of proposed approach.
\end{abstract}

Keywords-forensics; hard disk image; volatile memory dump; virtual machine; hibernation;

\section{INTRODUCTION}

Although digital forensics was recently challenged [1] it still remains the main way to investigate digital evidence and answer questions about previous digital states and events [2]. Digital forensics faces some challenges but is also area of intensive research and fast development to address those challenges.

Any forensics needs to follow strict procedures of evidence collection that ensures evidence admissibility in a court of law. Digital evidence is very sensitive and can be easily altered. Therefore procedures were developed that ensure integrity of the collected evidence. Initially, digital forensics used only static analysis that concentrated on extracting evidence from nonvolatile memory on media. Developments in digital world that include networking and encryption, as well as increased size of storage media required new methods. Live analysis was a next step. With live analysis data is collected from a running system. The idea is to gather data that is not available in a media-only forensic analysis, providing additional contextual information. In this process a content of the system volatile memory, RAM, provides valuable information. This opened another set of question on possible alterations to the system while collecting the evidence. The alteration might make collected evidence inacceptable. An alternative way to analyze content of the volatile memory is to capture it and examine it offline. The research of offline memory dump analysis is recent but very intensive one. In addition, virtualization techniques are finding its use in digital forensics. Booting copy of hard disk in a virtual machine enables obtaining some information that is not accessible by passive reading of data from the disk. It is possible to do this without altering copy of the disk. This opens some new possibilities and brings static and live analysis closer to each other.

This paper explores possibilities of combining offline hard disk and memory dump analysis. Virtual environment could enable starting OS from disk image without changing the image. Using data from memory dump could enable bringing the system close to the state it was in when it was seized. This provides for something similar to live response but in repeatable manner. It is important to state that the methods presented are proof of concept and are still not sufficiently robust to be considered ready for use by forensic analysts.

The rest of the paper is organized as follows. General issues of digital forensic analysis are addressed in section 2. Section 3 explains use of virtual machines for forensics. Volatile memory analysis is presented in section 4. Method proposed in this paper is explained in section 5. Results of testing of proposed method are given in section 6. Conclusion and discussion on directions for future research work are in section 7 .

\section{DIGITAL FORENSIC ANALYSIS}

Traditional approach to digital forensics is static analysis. This approach is most widely used, has established procedures and defined legal validity of evidence collected. In static analysis, forensically valid copy of all storage media of halted system is made. Tools for media analysis are then used to search for digital evidence. These tools are good at locating files and searching their content. File creation and modification times can be established. Deleted files usually can be recovered to some extent. Other interesting information like browsing history, email records and installed programs can also be recovered.

Static analysis has certain limitations. The biggest one being that it cannot provide complete picture of events. Recent work [3] explains main limiting factors.

In order to perform static analysis target system needs to be shutdown. This can be achieved with a proper shutdown 
sequence or simply by pulling a power plug. The second approach prevents possible execution of scripts set up to remove evidence in a case of the shutdown. It might also result in inconsistent disk state and data in write cache unsynchronized. In both cases of halting the target system its dynamic state is inevitably lost. Volatile memory content is not preserved, except for possible data paged to disk. Other relevant dynamic data like a process list, open network ports, established network connections and installed kernel modules cannot be examined with static analysis. Since all above items could be relevant for the investigation being conducted, static analysis gives incomplete evidence.

Encryption makes access to data stored in encrypted files or volumes much harder if not impossible. Encryption keys, used during system operation for access to encrypted data, are inaccessible once the system is powered off.

There is also a question if creation of an image for analysis is practical or even possible. For terabytes of disk data imaging can take many hours. Imaging of large RAID arrays, NAS, SANs is extremely difficult. The time and effort needed for analysis increases with the disk size. Since manual analysis is not feasible, automatic analysis is used. For terabytes of data even automatic analysis takes time [4] and requires a distributed analysis approach [5].

An often neglected drawback of static analysis is an inconvenience for a regular user. A system cannot be used while forensic copies of media are made. For certain systems that require high availability this could completely prevent static analysis.

An alternative to static analysis, or to be more precise complementary approach, is live analysis. In this case evidence is collected while the system is running. Live analysis resolves some of the issues of static analysis. On the other hand there are some open questions for live analysis. The most important issue is that with live analysis any action the analyst executes causes irreversible change of the investigated system state [6]. The change of the system state is against accepted principles in forensics that evidence should not be altered and might cause examination results not to be verifiable and repeatable [7]. Limitations imposed on acceptability of evidence collected by live analysis depend on the applicable legal system.

There are some other issues with live analysis. Investigator might not have appropriate level of access to the investigated system. In a case of a compromised system its integrity is questionable. An attacker might have modified the system in a way that prevents detection of attack and modifications. This is especially true if the investigated system's user interface is used. Even using known-good binaries from CD/DVD or other trusted media might not reveal the true facts since many of the forensic tools relay on the data provided by the kernel or the file system that might have been tampered with.

There are some alternatives to static and live analysis. Two that are relevant to this paper will be explained in more detail in the next section. The two are: static analysis using virtualization and offline analysis of volatile memory.

\section{VIRTUAL MACHINES FOR STATIC ANALYSIS}

Static analysis of hard disks is activity with a number of constraints. A basic requirement that no changes can be made to the original hard disk might significantly limit investigation efforts. Practice is to obtain forensic image of the original hard disk. There is a requirement for the image to remain unchanged to be acceptable as evidence in a court of law. Some information from the hard disk or its image cannot be obtained by passive reading of the data from the disk. Sometimes an operating system and/or programs from the disk need to be started in order to get insight into what is on the disk and what it was used for. Starting the program or the operating system from the disk unavoidably includes writing to the disk. This writing renders the disk unusable as evidence.

Usage of virtual machines was proposed for an analysis phase of a digital forensic investigation [8]. The idea is to create a virtual machine from an image to be investigated. Since the virtual machine simulates only some basic hardware components the image cannot be immediately booted in the virtual machine. There are many changes to the original environment required to enable the image to boot in the VM environment, and once the system is booted new data will be written to the original image thus modifying it. Such a changed image would be immediately challenged in a court of law as flawed.

This issue was addressed by CERT. They created Live View [9]. Live View is a forensics tool that creates a VMware virtual machine out of a raw disk image or a physical disk. This allows the forensic examiner to boot up the image or the disk and gain an interactive, user-level perspective of the environment, all without modifying the underlying image or disk. All changes made to the disk are written to a separate file. This enables continuation of analysis from where it stopped or a restart from the original state of the image or the disk.

\section{VOLATILE MEMORY ANALYSIS}

A recent addition to live analysis is an idea to make a dump of a volatile memory for offline analysis. Volatile memory analysis shows promise in that the only source of evidence is a physical memory dump. An investigator can then build the case by analyzing the memory dump in an isolated environment that is non-obtrusive to the evidence. This approach addresses some of the issues with live analysis. It limits impact to the compromised system, the analysis is repeatable and it is possible to ask new questions later. Also, offline volatile memory analysis does not rely on operating system of possibly compromised machine. This enables detection of processes hidden by installed rootkit or a similar tool [10].

The first idea was to use a special pre installed hardware that can copy memory to an external storage device without modifying its contents [11]. The need for the special hardware that must be fitted to the system being protected before anything bad happens makes this approach impracticable in general case, but it had started a lot of research dedicated to analysis of memory dumps. a number of papers is devoted to Windows memory analysis [12][13][14][15]. A method for recovering files mapped in a memory and to link mapped file information process data is subject of [16]. Different tools for 
memory analysis are proposed like: FATKit [17], BodySnatcher [18], Volatools [19] and FACE [20].

The biggest issue with the memory dump analysis was a lack of tools. This is because with every release of a new operating system, the physical memory structure changes. In spite of difficulties some tools are being developed. The first ones had only basic functionality [21][22]. Current tools [23][24] are still script based but offer functionality similar to the live analysis. They allow an investigator to interrogate an image in a style similar to that used during a live response.

The most recent development in volatile memory analysis is usage of hibernation. Most current computers systems and OS have power management features that save the state of the computer while the processor and devices (hard drive, monitor, etc.) are disabled to conserve power. This is also known as suspending to disk feature. If the system state and memory are copied to disk using the power management features of the computer, then this method may be more reliable than the software solutions for creation of memory dump [11]. System state and memory are usually saved in a file that contains all the physical memory saved by the OS and aims to be restored by the user the next time the computer is powered on.

Live forensics analysis is used on a physical memory dump to recover information from a targeted machine. One of the main problems is to obtain a readable physical memory dump, hibernation is an efficient way to save and load physical memory. Hibernation analysis has notable advantages. System activity is totally frozen, therefore coherent data is acquired and no software tool is able to block the analysis. The system is left perfectly functional after analysis, with no side effects.

Usage of hibernation was mentioned for the first time when volatile memory investigation was suggested [11]. It was not really used until recently when a tool for Windows hibernation file analysis was presented [25].

\section{PROPOSED METHOD}

This paper proposes combination of static and live analysis. The combination should provide more insight into current state of the system being examined and better understanding of the events that led to it.

A hard disk image can be booted in VMware using Live View. The memory dump obtained before the system was powered off can be used to restore the system booted in virtual environment to the state it was in when the memory dump was created or as close to it as possible. The memory dump contains data on the processes that were running on the system. It also contains times when the processes were started. This enables manual start of the programs that were not started during boot process. The programs can be started in the same order as they were started on the investigated system. This includes hidden programs like rootkits that would not be visible in live analysis. Open files and open network ports with processes that opened them are also stored in the volatile memory dump. With this information investigator can try to open the same files and network ports. Furthermore, sometimes even encryption keys can be recovered from the memory dump
[19], but this is out of the scope of this paper and will not be tested.

Since Live View enables booting of an image in VMware while keeping the image file intact, an investigator can use trial and error approach trying to restore and understand the events that took place on the system. What-if analysis can be performed with the goal to understand possible development scenarios. The best thing is that investigators actions are repeatable and can be presented in a court of law.

The proposed approach to live analysis is to simply put the system to hibernation. This is a fast way to preserve volatile memory and system state with no need for additional tools and no change of system state. After system goes to hibernation, hard disk image can be created for further investigation. The image contains hibernation file that in turn contains volatile memory content.

As a proof of concept, test system was created and was taken through the proposed steps of acquisition and analysis. Testing results are presented in the next section.

\section{TESTING}

System to be investigated was Windows XP SP3 installed on 8 GB NTFS partition with $256 \mathrm{MB}$ of RAM. The hard disk partition and RAM are small compared to the current standards. They are set small to enable a faster manipulation but big enough to show the procedure. A user with administrative privileges was logged on the system. Some sample programs were stared on the system. One file was opened for editing in Notepad. Netcat tool was started from a command prompt and set to listen for incoming connections on port 80. TrueCrypt 6.1 a [26] was used to create a new volume as an encrypted file container. One file on this encrypted volume was opened for editing in Notepad.

At this point the system was hibernated. The hard disk was taken out of the computer. An image of $8 \mathrm{~GB}$ partition in a raw (dd) format was created using dd tool. This image was stored on the investigators desktop computer and set read only. MD5 hash value was calculated using Microsoft File Checksum Integrity Verifier. The value was stored for later comparison.

Live View $0.7 \mathrm{~b}$ was started and given some basic data about the image and the environment it should be booted in. It was instructed to provide the same amount of RAM, $256 \mathrm{MB}$, in the virtual machine. Also, information that the image is Windows XP was supplied. Live View created necessary files in the selected directory. Than Live View started VMware Server 1.0 .8 and pointed it to virtual machine files it created from the image.

VMware server started boot up process. It realized that the system was hibernated and tried to wake it up. Coming out of hibernation failed, which was expected due to hardware difference between the original system and virtual environment. Windows XP offered to delete restoration data and boot up system without retrieving hibernated system state. It was accepted and the system started. The programs that were manually started on the original system before hibernation were not running. The same goes for the files manually opened 
on the original system. There was a message about new hardware being found, but it was ignored at this point. The system in virtual machine was powered off. It was done for two reasons. One was to test if the image has changed. Image, being read only, has not changed which was confirmed calculating its MD5 hash again. The other reason for shutdown was to extract hibernation file from the image.

Partition image was mounted, read only, using Mount Image Pro v3. File hiberfil.sys was copied from the mounted image to the file system. The copy was set read only and its MD5 hash value calculated. Using SandManSHELL utility [27] memory dump was created from the hiberfil.sys. Again the file was set to be read only and its MD5 hash was calculated. Although SandMan provides some basic utilities for extracting data from hibernation file they are rather crude. At this time it was concluded that it is easier and less error prone to use more mature tools for memory dump analysis, like Volatility framework [23].

Before analysis of the memory dump partition image was booted again using Live View. Live View offered choice to continue working on the modified virtual machine or start over from the image file. The option to continue working was selected. The system booted up without messages and without hibernated data. Now, there was a running picture of investigated system that could be compared with a picture of the system preserved in hibernation file.

Using Volatility it was possible to read various data from the memory dump. List of running processes with times when they were started was generated (Fig. 1). The list was compared with the list of running processes in virtual machine (Fig. 2). The difference was in the processes that were manually started on the original investigated system. Volatility list of DLLs loaded for each process (Fig. 3) show the command line, e.g. path, which was used to start the process.

For instance, the following lines show that Notepad was started with an empty file:

notepad.exe pid: 768

Command line : "C: $\backslash$ WINDOWS $\backslash$ system $32 \backslash$ notepad.exe"

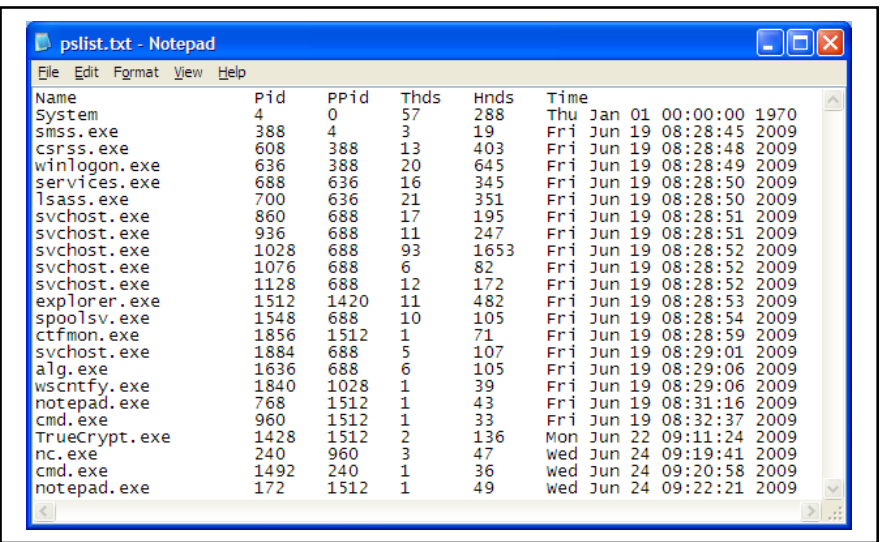

Figure 1. List of running processes from memory dump

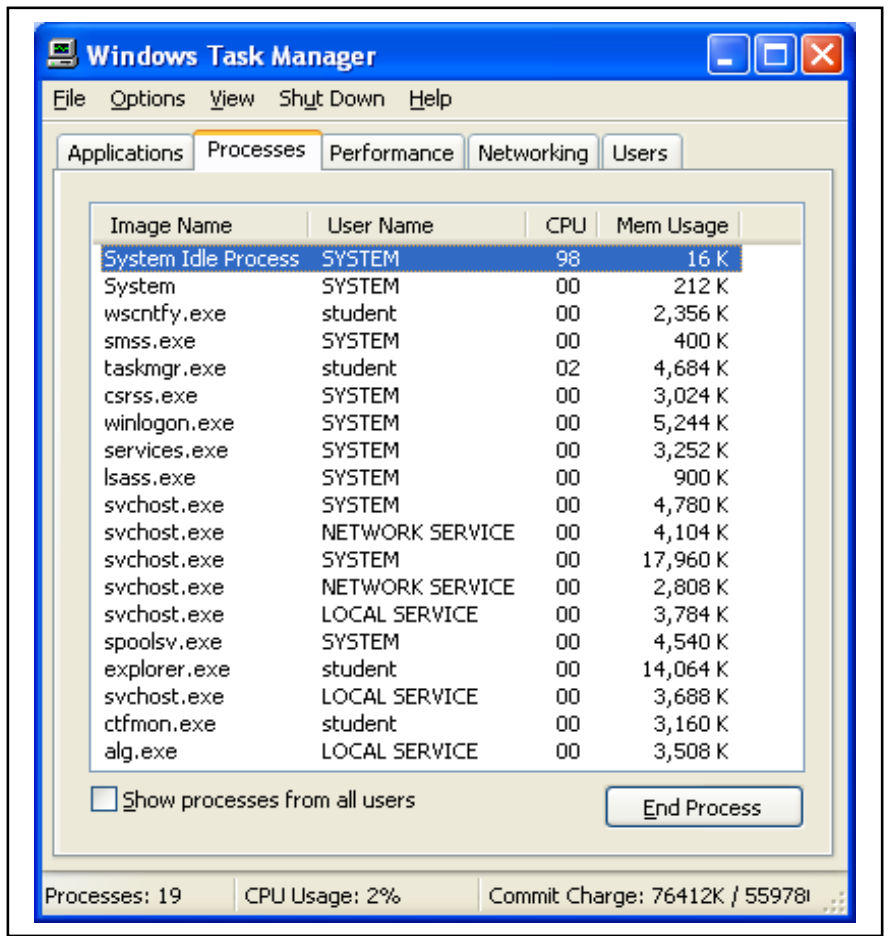

Figure 2. List of running processes in virtual machine list.

Other interesting things could have been noticed from this

Truecrypt.exe pid: 1428

Command line : "D: \TC $\backslash$ TrueCrypt.exe"

Above lines show that TrueCrypt was running on the system and that encryption was probably being used. Also, TrueCrypt was not started from the system disk but from some other volume. Since there is only one partition on the system it must have been a removable disk. This was exactly the case on the original investigated system.

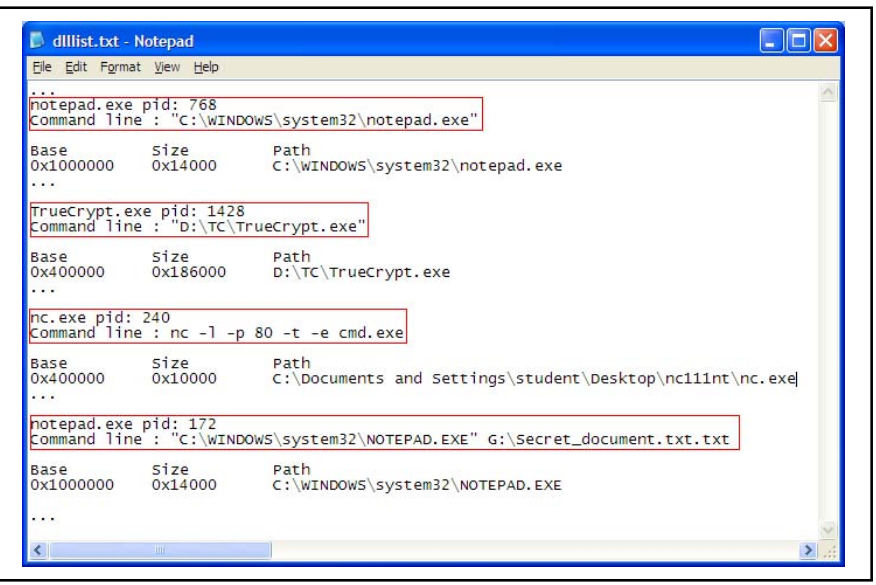

Figure 3. Parts of list of DLLs loaded for each processes 


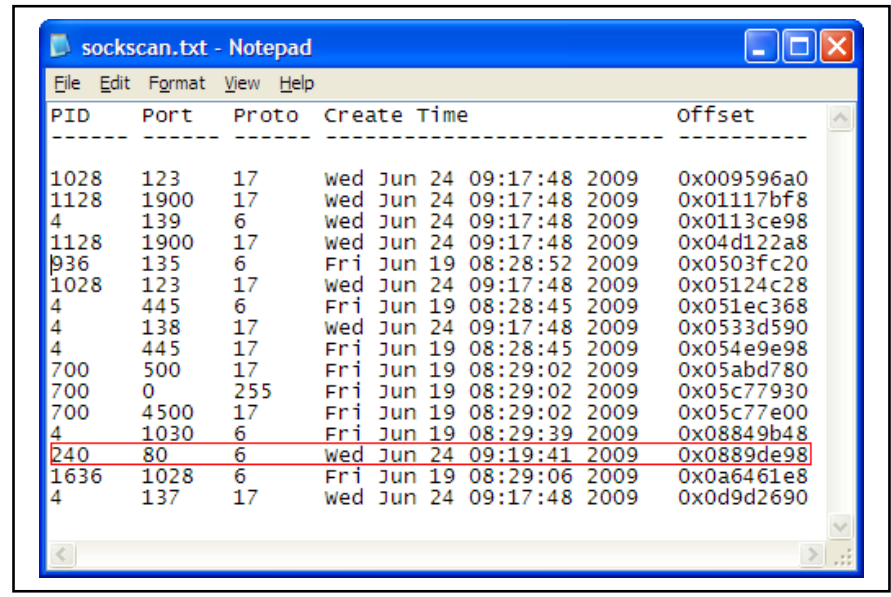

Figure 4. List of open sockets

Another two lines point to a different event:

nc.exe pid: 240

Command line : nc - - -p 80 -t -e cmd.exe

Netcat was started to listen on port 80 and set to spawn the shell when it gets connected to by a client. It could be confirmed from the Volatility scan of open sockets (Fig. 4):

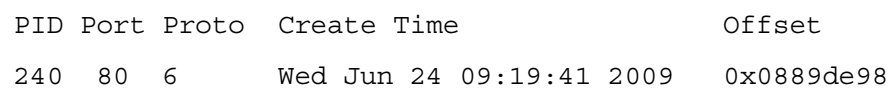

It can also be noticed, from Volatility list of running processes that Netcat has indeed received a connection and has spawned a shell:

$\begin{array}{lllllllll}\text { Name Pid } & \text { PPid } & \text { Thds Hnds } & \text { Time } & & \\ \text { nc.exe } & 240 & 960 & 3 & 47 & \text { Wed Jun } 24 & 09: 19: 41 & 2009 \\ \text { cmd.exe } & 1492 & 240 & 1 & 36 & \text { Wed Jun } 24 & 09: 20: 58 & 2009\end{array}$

Unfortunately, Volatility list of open connections was empty. It could have been expected since going to hibernation all open network connections had to be closed. This is something to keep in mind if using hibernation to preserve system state. It seems that running netstat and saving its output to a file should be a step before hibernation. In this way list of active connections of investigated system could be preserved.

Another interesting thing was noticed in the list of loaded DLLs. One instance of Notepad was started on an existing file:

notepad.exe pid: 172

Command line : "C: \WINDOWS $\backslash$ system32 $\backslash$ NOTEPAD.EXE" G: \Secret_document.txt.txt

File is located on a volume named G. Using Windows Sysinternals utility Strings [28] memory dump was searched for references to "G: $\backslash$ ". Results were saved to a file. This file, which provides (memory offset: string) mappings was used as an input to Volatility function strings. The output of this function is process that corresponds to these mappings (Fig. 5). Several similar entries that connect volume G: $\$ with TrueCrypt process (1428) were found.

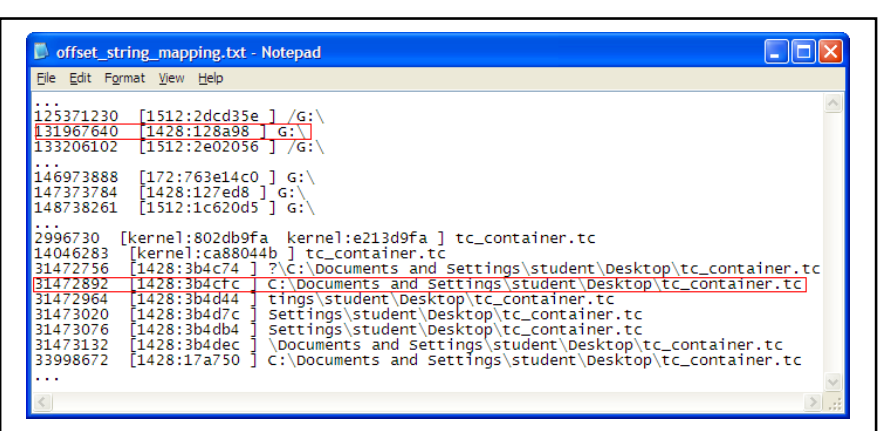

Figure 5. Part of memory offset:string mappings

Using the same utilities connections between TrueCrypt process (1428) and a file on a desktop tc_container.tc were found in the memory dump.

$31472892 \quad[1428: 3 \mathrm{~b} 4 \mathrm{CfC}] \quad \mathrm{C}: \backslash$ Documents and Settings $\backslash$ student $\backslash$ Desktop $\backslash$ tc_container.tc

The file tc_container.tc represents TrueCrypt encrypted file container. Next step would be to search the memory dump file for cached passwords and encryption keys as it was suggested in [19]. As it was previously stated decryption using memory content is out of scope of this paper and was not tried.

Above processes, found in the volatile memory copy from the original system, were started in the virtual machine. Similarly, the opened files found in the memory copy were open with the same applications in the virtual machine. No new information was obtained but the virtual machine was brought close to the state the original system was in before hibernation. If need be, this would enable further analysis of relationships among processes and files on the system.

After testing was over the virtual machine was stopped. All relevant files: the image of the original hard disk partition, hiberfil.sys and the memory dump created from it were checked for changes. Hash values for all three of them had the same values as for the original files. The system state has not changed, meaning that evidence was not altered. Also, the investigation process and steps taken are verifiable and repeatable. Accepted principles in forensics were not violated.

\section{CONCLUSION AND FUTURE WORK}

The proposed combination of static and live digital forensic analysis in a virtual environment offers new possibilities. Investigators can now play with a system image and volatile memory data trying to figure out the exact state of the original investigated system and sequence of events that led to it. All advantages of static analysis are still there. In addition, data usually available only during live analysis is on hand. Even process hidden from live analysis, like rootkits, are visible thanks to volatile memory copy. Virtual environment enables booting the image up for interactive investigation similar to live analysis. The best thing is that the original image is preserved and unchanged and that analysis is repeatable. This should make evidence obtained by using this approach acceptable in a court of law. It has to be repeated that this is 
still research and proposed approach should be thoroughly tested before real world usage.

There are some ideas on future usage and improvements. One usage could be to put a virtual copy of the system online with the same IP address and open network ports acting as a honeypot. Idea is to attract the same attackers that compromised the machine for the first time trying to locate them more precisely.

It would be interesting to establish with authority what are the changes made to the system in preparation for hibernation apart from closing network connection. Future work will also be directed towards trying to enable return from hibernation in virtual environment. This could be achieved by adjusting hibernation file for different hardware environment offered by virtual machine. SandMan project started work on writing to hibernation file. Another approach to this issue could be to copy data from hibernation file to snapshot file of virtual machine. Both files contain picture of the memory plus some additional info. It should be possible to convert one format to the other.

\section{REFERENCES}

[1] M.A. Caloyannides, "Forensics Is So Yesterday," IEEE Security and Privacy, vol. 7, Mar. 2009, pp. 18-25.

[2] B.D. Carrier, "Digital Forensics Works," IEEE Security and Privacy, vol. 7, Mar. 2009, pp. 26-29.

[3] B. Hay, M. Bishop, and K. Nance, "Live Analysis: Progress and Challenges," IEEE Security and Privacy, vol. 7, Mar. 2009, pp. 3037.

[4] V. Roussev and G.G. Richard III, "Breaking the performance wall: The case for distributed digital forensics," Proceedings of the 2004 Digital Forensics Research Workshop, 2004, pp. 75-82.

[5] I.I.I. Golden G. Richard and V. Roussev, "Next-generation digital forensics," Commun. ACM, vol. 49, 2006, pp. 76-80.

[6] F. Adelstein, "Live forensics: diagnosing your system without killing it first," Commun. ACM, vol. 49, 2006, pp. 63-66.

[7] M.M. Pollitt, "Principles, practices, and procedures: an approach to standards in computer forensics," Second International Conference on Computer Evidence, 1995, pp. 10-15.

[8] D. Bem and E. Huebner, "Computer forensic analysis in a virtual environment," International Journal of Digital Evidence, vol. 6, 2007.

[9] CERT, "Live View," http://liveview.sourceforge.net/, Jun. 2009.

[10] C. Waits, J.A. Akinyele, R. Nolan, and L. Rogers, Computer Forensics: Results of Live Response Inquiry vs. Memory Image Analysis, CERT, 2008.
[11] B.D. Carrier and J. Grand, "A hardware-based memory acquisition procedure for digital investigations," Digital Investigation, vol. 1, 2004, pp. 50-60.

[12] A. Schuster, "Searching for processes and threads in Microsoft Windows memory dumps," Digital Investigation, vol. 3, Sep. 2006, pp. 10-16.

[13] A. Schuster, "Pool allocations as an information source in Windows memory forensics," International conference on IT-incident management and IT-forensics, 2006, pp. 104-115.

[14] J.D. Kornblum, "Using every part of the buffalo in Windows memory analysis," Digital Investigation, vol. 4, Mar. 2007, pp. 24-29.

[15] A. Schuster, "The impact of Microsoft Windows pool allocation strategies on memory forensics," Digital Investigation, vol. 5, 2008, pp. 58-64.

[16] R.B. Van Baar, W. Alink, and A.R. Van Ballegooij, "Forensic memory analysis: Files mapped in memory," Digital Investigation, vol. 5, 2008, pp. 52-57.

[17] N.L. Petroni, Jr, A. Walters, T. Fraser, and W.A. Arbaugh, "FATKit: A framework for the extraction and analysis of digital forensic data from volatile system memory," Digital Investigation, vol. 3, Dec. 2006, pp. 197-210.

[18] B. Shatz, "BodySnatcher: Towards reliable volatile memory acquisition by software," Digital Investigation, vol. 4, Sep. 2007, pp. 126-134.

[19] A. Walters, N.L. Petroni Jr, and I. Komoku, "Volatools: integrating volatile memory forensics into the digital investigation process," Black Hat DC, vol. 2007, 2007.

[20] A. Case, A. Cristina, L. Marziale, G.G. Richard, and V. Roussev, "FACE: Automated digital evidence discovery and correlation," Digital Investigation, vol. 5, 2008, pp. 65-75.

[21] C. Betz, "memparser," 2005 , http://www.dfrws.org/2005/challenge/memparser.shtml, (accessed Jul. 2009)

[22] G.J. "kntlist," 2005 , http://www.dfrws.org/2005/challenge/kntlist.shtml, (accessed Jul. 2009)

[23] Volatile Systems, "The Volatility Framework: Volatile memory artifact extraction utility framework," https://www.volatilesystems.com/default/volatility/, (accessed Jun. 2009)

[24] A. Schuster , "Memory analysis: "PTFinder Version 0.3.05"," http://computer.forensikblog.delen/2007/11/ptfinder_0_3_05.html, (accessed Jul. 2009)

[25] N. Ruff and M. Suiche, "Enter Sandman (why you should never go to sleep)," PacSec applied security conference, 2007.

[26] T.C. Foundation, "TrueCrypt-Free Open-Source On-the-fly Encryption," http://www.truecrypt.org/, (accessed Jul. 2009)

[27] M. Suiche, "SandManSHELL Project," http://www.msuiche.net/hibrshell/, (accessed Jul. 2009)

[28] M. Russinovich, “ Strings - Windows Sysinternals," http://technet.microsoft.com/en-us/sysinternals/bb897439.aspx, (accessed Jul. 2009) 